Karl Werner Bübler: Presse und Protestantismus in der Weimarer Republik. Kräfte und Krisen evangelischer Publizistik. Witten 1970 (Luther-Verlag, Reihe "Evangelische Presseforschung" Bd. 1). 182 Seiten.

Zweierlei will diese neue religionspublizistische Arbeit leisten: zum einen, die Geschichte evangelischer Presseethik bis 1933 nachzeichnen und die Erzeugnisse aus jener Blütezeit protestantischen Presseschaffens durchleuchten; zum anderen, das bis heute zu beobachtende Mißtrauen der evangelischen Kirchen gegenüber den modernen Massenmedien als ein gewachsenes und historisch begründetes aufdecken.

Die Entwidklung einer Presseethik - von den Anfängen in Kaspar Stielers „Zeitungslust und Nutz" bis hin zur Situation in der Weimarer Republik - wird anhand wichtiger historischer Ereignisse und theoretischer Uberlegungen (Stieler, Bahrd, Hinderer u. a.) ausführlich dargestellt, wobei besonders die speziellen Motive einer evangelischen Presseethik berüdksichtigt werden.

Man hätte sich dabei gewünscht, daß das im Text sichtbare geschichtliche Fortschreiten schon in der Gliederung klarer zum Ausdruck gekommen wäre; wie überhaupt diese Gliederung wenig informativ ist, da die Schlagworte erst im Kontext verständlich werden. Die Abhandlung selbst führt indes schlüssig zu den Schaltstellen des weitgehend rückwärtsgewandten Presseverständnisses der evangelischen Kirchen und zu den Positionen, die die Situation der kirchlichen Presse in der Weimarer Republik kennzeichnen.

Auf diesem Hintergrund unternimmt der Verfasser den verdienstvollen Versuch, das vorhandene Material - gegliedert in Sonntagsblatt, Zeitungsandacht und religiöse Kurzgeschichte - zu sichten und die Wandlung der jeweiligen Gattung historisch zu verfolgen. Ein nicht unwesentliches Nebenprodukt dieser sorgfältigen Materialsichtung ist eine umfangreiche Bibliographie. Das Be- mühen jedoch, die Ergebnisse durch ausführliche Belegstellen abzusichern, geht dabei zu Lasten der Obersichtlichkeit - ein Dilemma, vor das freilich immer derjenige gestellt wird, der, wie im vorliegenden Fall, Neuland betritt. Der abschließende Teil "Theologie und Bewußtseins-Industrie" führt über den Untersuchungszeitraum hinaus und zeigt auf dem Hintergrund der dargestellten Tradition die heutigen Aufgaben einer christlichen Sozialethik auf. Der Weg, der die Kirchen aus ihrer "medialen Sackgasse" herausführen soll, liegt damit in der "thematischen und medialen Herausbildung von ,Teilchristentümern', weil nicht durch jedes Medium jeder mögliche Teilaspekt des Evangeliums vermittelt werden kann."

Eine Arbeit, die die Diskussion um das Problem "Kirche und Massenmedien" weiterführt.

J. Scheibe

Wilhelm Heinen (Hrsg.): Bild - Wort Symbol in der Theologie. Würzburg 1969 (Echter-Verlag). 308 Seiten.

Wenn es im einleitenden Bericht dieser $\mathrm{Zu}-$ sammenfassung eines Vorlesungszyklus der Katholisch-Theologischen Fakultät Münster heißt, daß Bild, Wort und Symbol zur Entfaltung des Menschen notwendig sind, dann ist dieser Beitrag der Theologie deshalb so bemerkenswert, weil theologische Diskussionen heute im Trend der Entmythologisierung zur Entsagung von redundanten Darstellungsformen auffordern und sich in Aussagelosigkeit verlieren, die durch soziales Engagement eigentlich nur noch im psychologischen Sinne "kompensiert" werden. Wilhelm Heinen deutet die Dichte der Bild- und Symbolgehalte für den technisch vereinnahmten und in Abkapselung sich identifizierenden Menschen als therapeutisches Element für eine „Aussöhnung des Menschen mit dem Menschen und mit Gott" (S. 20). Daß dabei 
anthropologische, tiefen- und strukturpsychologische Auffassungen Gefahr laufen, die heilsgeschichtliche Dimension der existentiellen Not des Menschen zu verkennen, ist ein Problem aller die Theologie stützenden $\mathrm{Hu}$ manwissenschaften geblieben. Dennoch beläßt gerade die Menschwerdung der christlichen Religion wie keiner anderen eine anthropologische Basis (Bernhard Kötting, vgl. bes. S. $103 \mathrm{ff}$.). In diesem Sinne ist die jüdische Apokalyptik mehr als nur ein literarischer Beitrag, sie stellt die das Christentum antizipierende Möglichkeit des Symbols dar (Josef Schreiner). In der Interpretation des christlichen Gleichnisses findet Joachim Gnilka die immanente Notwendigkeit verdichteter Bildsprache für das Wort Gottes: „Die positive Entscheidung für das Wort soll erreicht werden durch Zustimmung" (S. 102).

Die publizistische Reaktion auf das Wort Gottes liegt nicht in analytischer, zur Gegendeutung herausfordernder Verbalisation, sondern in der Inszenierung eines ganzheitlichen Begegnungsverhältnisses. Die Chance theologischer Ausdruckskraft entbindet sich nicht von den Extremen des intellektualistischen Nominalismus und der verdinglichten Bilderverehrung, sondern jenseits eines programmatischen Kalküls von der von Paul Tillich geforderten übergreifenden "Gestaltwerdung durch Sakramente und Symbole ${ }^{\alpha}$ (Erwin Iserloh, S. 137).

Karl Rahner sagt: „Wir müssen den Streit zwischen Sehen und Hören aushalten. Er, der Streit selber, ist die Anwesenheit des Friedens, der in Gott ist, gerade weil er die

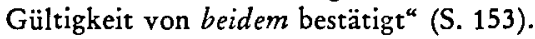

Die emotionale Unsicherheit des Menschen unserer Zeit ist wahrscheinlich weniger durch psychotherapeutisch-regressive Symbol, noch durch kulturpessimistische Autoritätssehnsucht (der Heinen anheimfällt), sondern durch sakramentale Erneuerung zu heilen (Peter Lengsfeld), die selbstbewußte Priester zu initiieren haben. Nicht umsonst kommt Wilhelm Weber zum Ergebnis: "Sein (des Priesters) pastorales Wirken wird durch so zahlreiche Normen und Vorschriften determiniert, daß er zumeist als ausführendes Organ eines prädeterminierten Systems erscheint ${ }^{*}$ (S. 266). Gegen diese autoritäre Vereinnahmung der priesterlichen Handlung, die zuletzt eine Verwaltung des symbolischen Aktes mit sich bringen muß, wendet sich Wilhelm Weber auf soziologischer Ebene (wie Peter Lengsfeld auf theologischer), wenn er behauptet: n. . . es ist eine Tatsache, daß heute derjenige dem Menschen am besten dient, der ihm am meisten $\mathrm{zu}$ bieten hat: der fähige Arzt, der fähige Ingenieur, der fähige Politiker und nicht zuletzt der fähige Priester. Professionelle Unterentwicklung darf nicht mit der ,Torheit des Kreuzes' kaschiert wer$\operatorname{den}^{\alpha}$ (S. 268). Der so geforderte Priester wird symbolisches Handeln nicht ritualisieren, d.h. zur eigenen oder des Amtes Befriedigung hochstilisieren wollen, sondern als primäre Verkündigung jeweils geschichtlich auffassen und in diesem Sinne Josef Glaziks These verifizieren können, daß der „wesentliche Unterschied zu den Symbolen der Völker gerade den offenbar machen, der zwischen Mythos und Geschichte besteht ${ }^{\alpha}$ (S. 283).

Wilhelm Dreier weist zum Schluß auf die Gefahr hin, daß Kirche sich heute durch "werbewirksame Worte" vereinnahmen läist, sich also in werbepsychologischer Anwendung befindet und ihrem nur sie kennzeichnenden paradoxen Auftrag entgeht, sich nämlich von einer Welt zu entbinden, der sie zu dienen hat. In diesen letzten Gedanken finden sich wichtige Ansätze $z u$ einer theologischen Publizistik, die sich nicht zu einer revolutionären Heilspropaganda umfunktionieren lassen darf; denn Kirche wird als Organisation Träger einer „unbequemen“ Gesinnung im bildnerischen, verbalen und symbolischen Dialog bleiben müssen, der auf die Zukünftigkeit des Heils verweist.

Das vorliegende Buch ist als Darstellung der publizistischen Potenz, die der (christlichen) Theologie innewohnt, geeignet, die Grundlage für eine Theologie der Kommunikation (außerhalb instinktresidualer Theorien) und in besonderem Sinne für ein funktionales System religiöser Publizistik zu bilden. Der religiöse Publizist wird in diesem Buch keine theologisch begründete Methodik seiner Arbeit finden können, jedoch wird der Auftrag, den er sich gestellt hat, in kommunikatorischimmanente Grundsätze der Verkündigung eingefaßt, die, ohne den Menschen in seiner Sinnlichkeit zu treffen, vergeblich sein muß.

P. Rech 
Martin Osterland: Gesellschaftsbilder in Filmen - Eine soziologische Untersuchung des Filmangebots der Jahre 1949 bis 1964. Stuttgart 1970 (Ferdinand Enke Verlag, Reihe "Göttinger Abhandlungen zur Soziologie ${ }^{\alpha}$, 19. Band). 253 Seiten.

Bei den in der Bundesrepublik in den Jahren zwischen 1949 und 1964 angebotenen Filmen besteht zwischen den Merkmalen "Handlungsrelevanz des Berufes im Film ${ }^{\text {" und }}$ „Entfremdungsgrad der Tätigkeit" ein $\mathrm{Zu}$ sammenhang: Der Beruf von Filmfiguren wird für die Filmhandlung zunehmend relevanter, wenn er einen individuell verfügbaren Dispositionsspielraum der Arbeit erlaubt und relativ frei von Subordination ist. Der in der industriellen Arbeitsorganisation entfremdete Mensch wird in den Filmen kaum dargestellt.

Im Bereich von Liebe und Ehe reproduzieren die Filme den Rückzug ins private Glück, das nur zeitweilig getrübt werden kann. Die Geschichte erscheint in den Filmen weitgehend als die von Kriegen, deren Unvermeidbarkeit unterstellt und deren Ursachen meist nicht genannt, geschweige denn diskutiert werden. Die weitaus meisten Filme (über 80 Prozent), die im 20. Jahrhundert spielen, sind manifest unpolitisch und entwerfen das Bild einer Gesellschaft ohne politische Probleme.

Das sind die wichtigsten Ergebnisse einer Inhaltsanalyse der Spielfilme in- und ausländischer Herkunft, die zwischen 1949 und 1964 in den Kinos der Bundesrepublik gelaufen sind. $\mathrm{Da} \beta$ diese Ergebnisse für denjenigen, der auch nur einen geringen Bruchteil des Verleihangebots in diesem Zeitraum aus eigener Anschauung kennt, kaum Uberraschungseffekte beinhalten, ist kein Argument gegen diese Arbeit, denn der wissenschaftliche Wert einer Untersuchung ist nicht abhängig von der "Neuheit", sondern lediglich von dem Grad der (bei aller subjektiven Implikation im Ansatz) intersubjektiven Absicherung ihrer Ergebnisse. Der Verfasser leistet diese Absicherung durch Anwendung der quantifizierenden inhaltsanalytischen Methode.

In Details sind allerdings Einwände gegen die Arbeit anzumerken: So werden häufig Merkmalszusammenhänge in den Filminhalten behauptet, ohne sie auf ihre Signifikanz hin zu überprüfen; an anderer Stelle klassifiziert der Verfasser das Gesamtfilmangebot nach bestimmten Gattungen, was zwangsläufig zu fragwürdigen Ergebnissen führt, da diese Gattungen nicht als Ausschlußkategorien zu definieren sind; neben der quantitativen Feststellung von inhaltlidhen Stereotypen im Gesamtangebot verliert sich der Verfasser häufig in der qualitativen Beschreibung von Einzelbeispielen, die nichts weiter besagt, als $\mathrm{da} ß$ es dies oder jenes Phänomen in dem einen oder anderen Film auch gibt, und dementsprechend unerheblich bleibt.

Trotz dieser Einwände ist Osterlands Studie vor allem für denjenigen, der sich medienpädagogisch mit dem Film befaßt, von grundsätzlichem Wert: Sie verweist in den nach der Reflex-Hypothese vorgenommenen präzisen Ergebnisinterpretationen exemplarisch auf den Zusammenhang zwischen Film und Gesellschaft und liefert damit ebenso wie andere Filmuntersuchungen ähnlicher Thematik Basismaterial für eine ideologiekritische Filmkunde, die nicht auf bloße innerästhetische Filmbegutachtung abzielt. Dazu tragen das Einleitungs- und das Schlußkapitel wesentlich bei: In der Einleitung wird eine knappe und übersichtliche Interpretation wichtiger Literatur zum Thema Film und Gesellschaft gegeben; der Schluß enthält generelle Hinweise auf die soziale Funktion des Films, wobei Funktion sowohl als gesellschaftliche Abhängigkeit als auch als gesellschaftliche Leistung verstanden wird.

P. Pleyer

Fritz Patrick Schaller: Zum Informationsrecht im kirchlichen Raum. Eine Studie nach den Texten des Vaticanum II. Freiburg/ Schweiz 1970 (Universitätsverlag, Reihe „Offentliche soziale Kommunikation“, Bd. 2). 134 Seiten.

Sch. faßt die Begriffe "Information" und "Informationsrecht" als anthropologische Begriffe, die als solche jeder Gesetzgebung vorgeordnet sind und somit Grundlage positiver Gesetze sein können (vgl. S. 13). Methodisch geht er von zwei Seiten her an die anthropologische Bestimmung dieser Begriffe heran: 1. von der Information als Funktion der menschlichen Person und 2. als Funktion der 
menschlichen Gesellschaft, wobei er jedoch betont, daß das Informationsrecht als soldhes nur „als einziges erkannt werden kann: als das Informationsrecht der sozialen menschlichen Persönlichkeit" (S. 36). Die nähere anthropologische Bestimmung soll innerhalb der Perspektiven der entsprechenden Texte des II. Vatikanum erörtert werden; dabei will Sch. die Zuordnung des Konzildekretes „Uber die Mittel der sozialen Kommunikation" zu den anderen Texten des Konzils in folgender Weise verstanden wissen: "Inter Mirifica" wird nur richtig interpretiert, wenn es in seinen Grundzügen als überholt betrachtet und im Lichte späterer Konzilstexte interpretiert wird" (S. 13). Mit dieser methodischen Vorentscheidung ist die Disposition der Studie gegeben. In einem ersten Kapitel schildert sie die Entstehung und Bedeutung von "Inter Mirifica" (S. 15-36); das zweite Kapitel „Information und menschliche Person" (S. 37-61) und das dritte Kapitel "Information und menschliche Gesellschaft" (S. 63-97) wollen die anthropologische Zielsetzung einlösen, während das vierte, als „Exkurs“ bezeichnete Kapitel, , ein mögliches Informationsrecht in der Kirche“ begründen will (S. 99-120).

Natürlich genügen die spärlichen Texte des Konzils, die sich unmittelbar auf die Information und das Informationsrecht beziehen, nicht, die vom Verfasser gestellte Aufgabe der Begründung eines Informationsrechtes im kirchlichen Raum zu erfüllen. Er behilft sich, indem er anthropologische Aussagen über Person und Gesellschaft aus den Konzilstexten (vor allem aus "Gaudium et spes") in die Erkenntnisse der Kommunikations- und Publizistikwissenschaften, die zugleich ihrerseits auf ihre anthropologischen Inhalte hin untersucht werden, einordnet. Wir halten dieses methodische Vorgehen grundsätzlich für legitim, nur müßte im einzelnen die Konsistenz publizistikwissenschaftlicher Begriffe nach ihrer Transposition auf eine anthropologische Ebene genauer und kritischer geprüft werden. Damit würde allerdings der Rahmen der vorliegenden Arbeit bei weitem gesprengt werden, da diese Konsistenzprüfung eine die verschiedenen wissenschaftlichen Disziplinen in sid integrierende Anthropologie voraussetzte. Ein sold integrierender anthropologischer Ansatz steht jedoch bis heute vor allem im Hinblick auf die Informations- und
Massenkommunikationsproblematik noch aus. Zwar bemüht sich Sh. in Einzelfragen um solch eine Vermittlung, so $z$. B. in der trefflichen Gegenüberstellung der Konstituanten des Informationsprozesses mit dem menshlichen Erkenntnisprozeß (S. 42-52); doch zu einem Gesamtentwurf einer „Informationsanthropologie" reicht dies nicht aus. Dazu bedarf es ebenfalls einer Fülle von Vorstudien, vor allem methodologischer Art, sowie einer Aufarbeitung der Relevanz kommunikationswissenschaftlicher Begriffe und Systeme für eine solche Anthropologie. Trotz des Verschweigens dieser grundsätzlichen Schwierigkeit ist der Mut und das Geschick, die den Verf. an eine anthropologische Bestimmung des Informationsrechtes herangehen ließen, anzuerkennen und zu bewundern.

So setzt auch unsere Kritik erst dort an, wo das Menschen- und Gesellschaftsbild des Konzils mit den Ergebnissen der Kommunikationsforschung derart verbunden werden, $\mathrm{da} \beta$ es scheint, als ob diese Verbindung so ohne weiteres möglich sei. Ein Beispiel: Sch. arbeitet sehr treffend die Hinordnung des Informationsrechtes auf die Wahrheit heraus (S. 57); es fehlt indes die Darstellung des Wahrheitsbegriffes, der hinter den Aussagen des Konzils steht. Statt dessen bietet Sch. selbst einige sehr schöne eigene Definitionen (S. $40 \mathrm{f}, 50 \mathrm{f}, 72$ ), die aber nicht unbesehen dem Konzil unterschoben werden dürfen. Mit einem unmittelbar aus den Konzilsdekreten eruierten Wahrheitsbegriff hätte Sch. unter Umständen insofern böse Überraschungen erleben können, als dieser dem Wahrheitsbegriff Pius' XII. - der auch von Sch. als unbrauchbar für eine Begründung des Informationsrechtes kritisiert wird (S. 32) - näher steht als dem demokratischen Verständnis von Wahrheit in einer pluralistischen Gesellschaft. Es fragt sich also, ob der Wahrheitsbegriff des Konzils in seiner massiven dogmatischen Akzentuierung die Information überhaupt noch unter dem Aspekt der Freiheit erfassen kann.

Von diesen Bedenken her scheint uns auch die Interpretation (S. 58) des Ausdrudss nwahre und gerechte Freiheit der Information" (Inter mirifica 12) durch Sch. zu optimistisch. Ferner bezieht er die Interpretation des Rechts auf Information „gemäß den Verhältnissen (condiciones) eines jeden" (In- 
ter mirifica 5) allzu naiv nur auf die Altersunterschiede des Publikums und somit auf die unbestrittene Notwendigkeit eines Jugendschutzes. Ein dogmatischer Wahrheitsbegriff (den der Rezensent dem Konzil allerdings unterstellen will) erlaubt jedoch darüber hinaus den zur Information verpflichteten Hierarchen und Politikern die Ausdehnung dieser "condiciones" auf die soziale und politische Stellung oder - im innerkirchlichen Bereich - auf die Position des einzelnen Gläubigen in der Hierarchiepyramide. Folglich könnten diese "condiciones" letztlich wieder zu einer Rechtfertigung des alten Geheimhaltungsprinzips führen (vgl. S. 12). Diese Befürchtung ist nicht von der Hand zu weisen, da das Konzil weder von den dogmatischen noch institutionell-organisatorischen hierarchischen Dimensionen eines Kirchenbegriffes abgegangen ist, dem eine vertikal-monologische Kommunikationsstruktur zugeordnet ist (vgl. das „Dekret über die Hirtenaufgabe der Bischöfe" ${ }^{\text {, }}$ sowie die berühmte „Nota praevia ${ }^{\star}$ !). Diese hierarchischen Dimensionen mit ihren vertikalen Anweisungs- und Kommunikationsmechanismen werden Sch. bei der Darlegung der Ergebnisse der Kommunikationsforschung leider nicht zum Problem. Er sieht nicht, daß diese vom Konzil bestätigten Strukturen auch die von ihm aufgezeigten Möglichkeiten eines Informationsrechtes in der Kirche (S. $100 \mathrm{ff}$ ) gefährden könnten.

Gerade die Uberspielung der Notwendigkeit von Strukturänderungen des instituierten Kommunikationssystems "Kirche“ ("das Maß des Menschen wird nicht an der Institution genommen, sondern an Christus“, S. 103) und dies trotz und angesichts der in diesem Punkte "hart bleibenden " Konzilstexte läßt eine Begründung des kirchlichen Informationsrechtes fraglich werden. Die Zuordnung von Hierarchie und „Volk Gottes" wird nur theologisch und nicht - was ebenso notwendig wäre - strukturell-juridisch beschrieben. Mit der Feststellung, daß ein Informationsrecht in der Kirche theologisch möglich ist, ist zwar eine anthropologische Gewißheit und sozio-kulturelle Uberzeugungsstereotype der modernen Gesellschaft einer durch die Jahrhunderte renitenten und unbußfertigen Kirche nahegebracht, aber auf welche Weise sich die theologische Anerkennung dieses Menschenrechtes auch auf den Sozialkörper
"Kirche" und das theologische Verständnis ihrer Organisationsgestalt (Ämterstruktur) auszuwirken hat, ist damit noch nicht $z$ wingend, verbindlich und effektiv festgelegt. Das heißt: das Informationsrecht ist theologisch wie kommunikationswissenschaftlich hinreichend anerkannt, nur: der theoretische Anspruch ist noch lange kein Unterpfand der praktischen Verwirklichung. Dazu ist nicht nur eine „Erhellung “ der Konzilsaussagen durch die Kommunikationsforschung oder eine "Verbindung" (S. 121) beider notwendig, sondern auch - und vielleicht vor allem - eine Kritik des Konzils durch die Kommunikationswissenschaften. Leider bleibt uns die vorliegende Studie diese Kritik schuldig. Ihr Verdienst besteht indes darin, daß sie zum ersten Mal überhaupt eine Gegenüberstellung anthropologischer Aussagen mit Ergebnissen der Kommunikationsforschung gewagt hat und auf eine Reihe theologischer Begriffe wie „Volk Gottes" (S. 100 ff), "Charisma" (S. $104 \mathrm{ff}$ ) und "Laien" (S. $107 \mathrm{ff}$ ) aufmerksam macht, die im Binnenraum der Kirche Verständnis für die anthropologische Grundkategorie "Informationsrecht" wecken könnten.

Eine Einzelkritik hätte u.a. die ungewöhnliche Verwendung des Begriffes "Soziusfunktion “ S. $64 \mathrm{ff}$ zu monieren, der in der Publizistikwissenschaft anders, spezieller, besetzt ist, als Sch. ihn verwendet. Er beschreibt unter diesem Begriff im Grunde das, was durch die Kategorie der Reziprozität oder aber durch den Interdependenzeffekt publizistischer Interaktionen treffender beschrieben wird. Ferner ist die Brauchbarkeit des gefühlsbeladenen Schlagwortes vom "familiären Austausch" S. 118 für eine Beschreibung der Kommunikationsstruktur der Kirche fragwürdig.

G. Deussen

José Maria de Vera: Educational Television in Japan. Tokio 1967 (Sophia University und Charles E. Tuttle Co., Tokio). 140 Seiten.

Mitoji Nishimoto: The Development of Educational Broadcasting in Japan. Tokio 1969 (Sophia University und Charles E. Tuttle Co., Tokio). 287 Seiten. 
Bedeutung und hoher Stand des Schul- und Bildungsfunks in Japan sind in den letzten Jahren durch entsprechende Preise für japanische Produktionen und noch mehr durch entsprechende Fachkonferenzen in Japan selbst immer mehr bekannt geworden. Dankbar ist man deswegen, zwei Werke eines japanischen Verlages anzeigen zu können, die sich gegenseitig ergänzen. Während das schon 1967 erschienene Werk von José Maria de Vera, Professor an der katholischen Sophia-Universität, mehr einen Bericht über den Begriff, Inhalt, Rezipientenschaft und Wirkungen des Bildungsfernsehens in Japan gibt (Stand etwa 1965), handelt es sich bei Mitoji Nishimoto um einen der Pioniere des japanischen Schulfunks, dessen ursprïnglich japanisches Buch ins Englische uibertragen wurde. Wie notwendig eine Beschäftigung mit dem Phänomen des japanischen Schul- und Bildungsfunks ist, wird deutlich, wenn man bei de Vera erfährt, daß schon 1964 täglich ein 8-Stunden-Schulfunkprogramm uiber das Fernsehen ausgestrahlt wurde, was von Nishimoto für 1968 so ergänzt wird, daß damals allein "Nippon Hoso Kyokai“ (NHK) Fernsehschulfunksendungen von 9 Uhr vormittags bis 4 Uhr nachmittags täglich mit einer halbstündigen Unterbrechung zur Mittagszeit ausstrahlte (de Vera S. 121; Nishimoto S. 102 f). Von nicht geringem Interesse dürften audh die Einwirkungen des japanischen Rundfunksystems auf den Schulfunk sein; denn neben der öffentlich-rechtlichen Anstalt "Nippon Hoso Kyokai" (NHK) gibt es seit 1951 auch kommerzielle Stationen in Japan. Die beiden 1965 neben der entsprechenden NHK-Abteilung auf Bildungs- und Schulfunksendungen spezialisierten Fernsehstationen haben sich bei Erteilung der entsprechenden Lizenz auf 50 Prozent des Programms als Schulsendungen (educational), 30 Prozent als Bildungssendungen (cultural) und 20 Prozent Unterhaltung festgelegt - so die Sendergruppe NET (Nippon Educational Television), die 1964 80 Prozent des Landes erreichte (de Vera S. $47 \mathrm{f}$ ). 60 Prozent des Programms als naturwissenschaftliche Bildungssendungen (science education), 15 Prozent als allgemeine Bildungssendungen (non scientific educational) und 25 Prozent als Nachrichtensendungen - strahlt der der „Japan Science Foundation " gehörende Kanal 12 in Tokio (de Vera S. 54) aus. Der besondere Wert von de Veras Veröffentlichung liegt in der klaren Gliederung und Darstellung aller bis 1965 vorhandenen Informationen und Untersuchungen zum Gesamtbereich Bildungsfernsehen in Japan, wobei viele nur in japanischer Sprache erschienene Studien (von 10 Seiten Literatur fast 8 in japanischer Sprache!) erstmalig auch der westlichen Welt in einer zusammenfassenden Darstellung bekannt werden.

Der Vorteil, aber auch die Schwäche von Nishimotos Veröffentlichungen liegt darin, $\mathrm{da}$ es sich hier um den Bericht eines Beteiligten handelt, der natürlich subjektive Meinungen und Ansichten einschließt. Liest man de Veras Buch zuerst, erfährt man dort, daß es sich bei Nishimoto um den Vertreter einer bestimmten Richtung in der Auseinandersetzung um die den Schul- und Bildungssendungen zugrunde liegenden $Z$ wecke und Ziele handelt (etwa in der Frage, wie weit Fernsehsendungen vom Lehrer begleitet oder eingeleitet werden müssen: vgl. de Vera S. 26-30). Die Darstellung Nishimotos ist sehr stark geschichtlich und aus seinem eigenen Erleben geprägt. Streckenweise für einen nichtjapanischen Leser etwas langatmig (z.B. S. $65 \mathrm{ff}$ ) und zum Teil weit ausholend, bietet sie aber doch eine Fülle von Einzeldaten und auch tabellarischen Ubersichten, die leider nicht immer quellenmäßig belegt werden. (Irgendwelche Hinweise auf ergänzende oder weiterführende Literatur fehlen völlig.) Die Studie Nishimotos umfaßt sowohl den Hör- als aud den Sehfunk, dessen Entstehung und Entwidklung ein eigenes Kapitel gewidmet ist (S. 91-112). Ein weiterer Abschnitt ist den Korrespondenzkursen in Verbindung mit dem Schul-, Hör- und Sehfunk (letzterer seit 1961) gewidmet, die in Japan für die verschiedenen Schularten unter Beteiligung führender Schulen in allen Teilen des Landes (vgl. z.B. S. 119) zu finden sind (Sendezeiten 1968: Hörfunk werktäglich 8 bis $11 \mathrm{Uhr}$; Sehfunk werktäglich 9-11.25 Uhr, sonntags 10-12 Uhr und 13.30-14.30 Uhr; S. $135 \mathrm{ff}$ ). Von einigem Interesse dürften kurze Zusammenfassungen von Forschungsergebnissen zu den Nutzungsbedingungen für den Schulfunk, Wirkungen, Schülerreaktionen, Programmwünsche, Methoden des Schulfunkempfangs und die Reaktionen der Lehrer sein (S. 151-167). Die wohl mehr für japanische Leser gedachte Uberschrift über den Schul- 
funk in außerjapanischen Ländern wäre für die englische Ausgabe des Werkes wohl besser auf die Darstellung der Entstehung und Entwicklung des Japan-Preises (ab 1965) und der internationalen Beziehungen des japanischen Schulfunks (speziell zu Entwidklungsländern) beschränkt worden (etwa S. 228-248). Nach den Erfahrungen und der Meinung Nishimotos ist Schulfunk mehr als nur eine Illustration für Textbücher in der Schule und auch mehr als nur ein willkommener Ersatz für solche Fächer oder Themen, die in Lehrbüchern nur schwer erklärt werden können. Nur der Schulfunk kann jene Differenz überwinden, die $z$ wischen dem herkömmlichen Lehrstoff der Lehrpläne und der sich ständig ändernden Welt unserer Tage besteht. Die Möglichkeiten des Funks hinsichtlich Schnelligkeit, Gleichzeitigkeit, Aktualität, Distanzüberwindung usw. müssen auch für die Erziehung nutzbar gemacht werden, auf daß sie sich zu einer auf Zukunft ausgerichteten Disziplin entwidkle. Dabei ist dann die internationale Zusammenarbeit (vgl. S. 259-267) zwischen den Schulfunk- und Bildungsfachleuten nur eine Selbstverständlichkeit.

\section{F. J. Eilers}

\section{KURZBESPRECHUNGEN}

Centrum voor Communicatie-Wetenschappen (Hrsg.): Krant en omroep: statuut en vrije berichtgeving. Referaten van het Derde Vlaams Congres voor Communicatiewetenschap. Löwen/Belgien 1971 (Selbstverlag des herausgebenden Instituts, B-3000 Löwen, E. Van Evenstraat 2 A). 103 Seiten.

Das Zentrum für Kommunikationswissenschaften an der Katholischen Universität Löwen legt den Bericht über den 3. Flämischen Kongreß für Kommunikationswissenschaft ror. Seine Problemstellung war den Auswirkungen von Kommunikationsverfassungen auf die freie Berichterstattung gewidmet. Der Band enthält die Einführungsreferate sowie Berichte aus den je drei Presse bzw. Rundfunk zugeordneten Arbeitsgruppen und aus der Plenumsdiskussion.
Enne Dreppenstedt: Der Zeitungs- und Zeitschriftenmarkt. Hamburg 1969 (Verlag für Buchmarkt-Forschung. Reihe: Schriften zur Buchmarkt-Forschung, Bd. 15). 309 Seiten.

Man erfährt, wie ein Teil des Monstrums ,Medienumwelt' (gemäß dem McLuhan'schen Schlagwort: 'the medium is the message'), das die Zeitgenossen heute - je nach Standpunkt - schreckt oder bedient -, von nahem gesehen, beschaffen ist. Es geht in diesem Werk, das - ursprünglich eine sehr fleißige und wohlgeordnete Dissertation - nun zum vorliegenden Buch überarbeitet wurde, um den Teil ,Publikumspresse', d.h. Zeitungen und Zeitschriften von allgemeiner Verbreitung und gewissem universalem Inhalt - im Gegensatz zu den Fach- und Kundenzeitschriften. Es wird freilich nur die Presse in der Bundesrepublik näherhin untersucht.

Das Buch konzentriert sich auf die Untersuchung der Druckergebnisse im Bereich des letzten Bedarfs, d.h. auf Aspekte wie Bezugsarten, Bedarfsgewohnheiten des Publikums, Bedarfsschwankungen, und setzt zum Zweck einer möglichst genauen Diagnostik den Röntgenapparat und das Seziermesser besonders genau im Bereich, Absatzwirtschaftliche Organe und Absatzwege' an (Unterteilung: Absatzorgane des Verlages und selbständige absatzwirtschaftliche Organe). Einleitend werden kurz Voraussetzungen gestreift, die ein Druckergebnis ermöglichen, wie gesicherte Ergebnisse der Medientheorie, Verlagsorganisation und juridische und betriebskundliche Aspekte. Das Buch schließt mit einer kurzen Ubersicht, wie weit die entsprechenden Organe ins Ausland und welche Organe umgekehrt vom Ausland her auf welche Weise nach Deutschland hineinwirken. Ein sehr konkreter Zug beherrscht das Werk, Statistiken (bis 1968) bilden geradezu sein Rüdkgrat. (So findet man z. B. Auskunft, in welcher Stellung der bundesdeutsche Konsument zu lesen pflegt - liegend, stehend, aufrecht sitzend, marschierend etc.). Auch die Frühgeschichte wird, wo nötig, mit einbezogen. Sehr aufschlußreich sind $\mathrm{Ab}$ schnitte, die sonst nur in esoterischen Fachzeitschriften zu finden sind, wie etwa Organisation, Entwicklung, Praktiken und Ertrag der Lesezirkel. Eine Zugabe bildet eine ausführliche Bibliographie. Allerdings hätte man sich noch eine Zusammenfassung des Stoffs 
in einem Sachregister wünschen können, doch ist auch das Inhaltsverzeichnis sehr ausführlich.

Alles in allem ein Werk, das besonders jenen kirchlichen Kreisen empfohlen werden kann, die konkret erfahren wollen (und müßten), um wie viel klüger die ,Kinder dieser Welt ${ }^{c}$ (etwa mit ihrer Boulevardpresse) wirklich waren und sind.

\section{K. Vospernik}

Joachim H. Knoll, Georg Wodraschke, Jürgen Hütber: Jugend und Kulturpolitik. Eine Untersuchung über die kulturpolitische Informiertheit und Meinung Jugendlicher in einer Großstadt des Ruhrgebiets. Neuwied/Berlin 1970 (Hermann Luchterhand Verlag, Reihe „Aktuelle Pädagogik “). 277 Seiten.

Die Autoren sind unzufrieden mit dem herkömmlichen Begriff Kulturpolitik, und doch stellt ihre Arbeit eine im weiteren Sinne kulturpolitische Forschungsleistung eher dar denn einen Beitrag zum (sozialempirisch fundierten) erziehungswissenschaftlichen Schrifttum. Ihr Hauptinteresse galt, wie sie selbst schreiben, folgenden Aspekten des kulturpolitischen Spektrums: Kulturföderalismus, Schulwesen und Schulreform, schulische und berufliche Aus- und Weiterbildung, staatliches Förderungswesen, Teilnahme am kulturellen Leben, Massenmedien und Medienkonsum. Die starke Bedeutung, die der „öffentlichen Kommunikation " eingeräumt wird, zeugt für eine wirklichkeitsnahe Einschätzung der Ausgangslage ebenso wie die repräsentative Auswahl von 495 Befragungspersonen aus der Grundgesamtheit eines künstlichen Geburtsjahrgangs 1949/50 einer Ruhrgebietsgroßstadt (Bochum). Zur Befragungszeit (1967/68) waren diese Jugendlichen im Normalfall in den Abgangsklassen der Real- oder Berufsschulen bzw. in der entsprechenden Gymnasialklasse. Sie wurden auf der Basis eines Fragebogens in Interviews befragt. Die Methoden sind sorgfältig dargestellt. (Man vermißt die Datierung der Interview-Phase.) Die Ergebnisse, die im einzelnen hier nicht referiert werden können, bieten, gerade auch im Hinblick auf die $\mathrm{Zu}$ wendung von Jugendlichen zu den Massenmedien, eine große Fülle von Material, das als Basis-Faktenwissen für schulische und außerschulische Jugendarbeit ebenso geeignet ist wie als Korrekturmittel gegenüber kulturkritisch gemeinten Teufelsbeschwörungen, wie sie einen guten Teil der populär-medienpädagogischen Literatur durchziehen. Einige Feststellungen aus der abschließenden $\mathrm{Zu}$ sammenfassung seien an den Schluß gestellt: Jugendliche lassen "Kultur" in der Rangfolge kommunaler Haushaltspolitik an letzter Stelle rangieren. Uber aktuelle Kultur-Ereignisse in der eigenen Region haben die Jugendlichen nur beiläufige Kenntnisse, umso bessere, je stärker sie sich Presse und Fernsehen zuwenden. Die Hälfte der siebzehnjährigen Großstadtjugend hat noch nie eine Theateraufführung besucht, 6 Prozent sind regelmäßige Theaterbesucher.

M. Scbmolke

Winfried B. Lerg: Das Gespräch. Theorie und Praxis der unvermittelten Kommunikation. Düsseldorf 1970 (Bertelsmann Universitätsverlag). 376 Seiten.

nGegenstand dieser Untersuchung ist die unvermittelte Kommunikation, sind die Gespräche von Angesicht zu Angesicht unter mindestens zwei Personen“ (S. 10), grenzt Winfried B. Lerg sein Thema $a b$, das er für seine Habilitation gewählt hat und das jetzt in Buchform vorliegt. Es geht ihm darum, "in Bericht und Kommentar" die „Entfaltung und den gegenwärtigen Stand des Wissens über grundlegende publizistische Verhaltensweisen darzustellen. Dabei geht es um die Auswertung sowohl der beschreibenden als auch der analytischen Forschungen verschiedener Disziplinen über den individuellen oder sozialen Austausch in seinen mannigfaltigen Formen der unterrichtenden, werbenden, orientierenden und unterhaltenden Gespräche“ (S. 10).

Der I. Teil (S. 35-59) beschäftigt sich mit der Praxis der unvermittelten Aussage, der II. Teil (S. 62-226) mit der Praxis des unvermittelten Austausches und der III. Teil (S. 227-321) mit der Theorie der unvermittelten Kommunikation. Der I. Teil untersucht das unsichere Zeugnis (S. 36-46) und den unsicheren Zeugen (S. 47-59), im II. Teil werden psychische (S. 62-87), soziale 
(S. 88-171) und publizistische Kommunikationsspannungen (S. 172-220) abgeklopft. Ein erster Befund (S. 221-226) schließt sich an. Der III. Teil, der sich mit der Theorie der unvermittelten Kommunikation beschäftigt, unterscheidet die entscheidenden (S. 228 bis 268) und die unsicheren (S. 269-281) Gespräche, stellt die Gerüchtschemata (S. 282 bis 313) vor und liefert einen letzten Befund (S. 134-321).

Der erste Befund (S. 221) trägt die Forschungsergebnisse zur Phänomenologie der unvermittelten Aussage und des unvermittelten Austausches vor. Kritisch faßt Lerg zusammen: "Journalisten, Kulturkritiker und einzelne Zeitungswissenschaftler - sie verkörpern ohnedies lange Zeit die beiden erstgenannten Rollen - haben Gerücht und Klatsch beobachtet und beschrieben, überwiegend als Moralisten politischer oder gesellschaftlicher Zustände. Belesene Studien sind dabei entstanden, kritische Aufsätze, pointierte Essays und jüngst noch eine Beichtspiegeldefinition, aber keine einzige wissenschaftliche Untersuchung" (S. 225/226).

Der letzte Befund (S. 134) faßt die Empirie zur unvermittelten Kommunikation zusammen und stellt Hypothesen zur Theorie menschlichen Gesprächverhaltens und seiner Merkmale auf (S. 315). Lergs verdienstvolle Arbeit hat mit Sicherheit ihr Ziel, „eine Bestandsaufnahme wichtiger und theorie-orientierter Grundannahmen über das Gespräch in der Gruppe und in der Gesellschaft" (S. 10) $\mathrm{zu}$ leisten, erreicht.

Das von ihm vorgetragene Forschungsergebnis macht aber deutlich, wie wenig auf diesem Gebiet bisher geleistet wurde (oder werden konnte) und wieviel Arbeit noch bewältigt werden muß.

\section{P. Becker}

Wolfgang Marhold: Fragende Kirche. Über Methode und Funktion kirchlicher Meinungsumfragen. München/Mainz 1971 (Chr. Kaiser Verlag, Matthias-Grünewald-Verlag, Reihe "Gesellschaft und Theologie“, Abteilung: Praxis der Kirche Nr. 5). 195 Seiten.

Diese „Befragung der Befragungen ${ }^{\alpha}$ ist im Raum der evangelischen Kirche angesiedelt. Marhold hat für ihren Bereich 115 Befra- gungen erfaßt, um zu erfahren, „welche Motivationen sich hinter diesen Befragungen verbergen, von welchen Konzeptionen die Befragungen bewußt oder unbewußt ausgehen, welche kirchliche Praxis sich hinter diesen Befragungen verbirgt" (S. 7, Vorwort Norbert Greinachers).

Der Autor definiert: „Der theoretische Ort unserer Untersuchung ist dort, wo die Theologie in Konfrontation mit den Problemen, die durch die gegenwärtige Selbstauslegung des Christenums in Lehre und Handeln erzeugt werden, die Unausweichlichkeit erfährt, eigene Fachgrenzen zu überschreiten, bisher unübliche Methoden zur Kenntnis zu nehmen und sich ihrer zu bedienen; und zwar mit dem Ziel, auf die Nöte, Bedürfnisse und Fragen der heutigen Menschen besser und funktionaler eingehen zu können " (S. 17).

Von drei Seiten her versucht Marhold sein Thema zu bearbeiten: Er beschreibt die Befragungen in ihrem Erscheinungsbild (Information), dann erläutert er Methoden, Absichten und Implikationen der Befragungen (Interpretation) und verbindet damit die Frage nadh der Lehre der Befragung. Dem schließt sich die Frage nach der Theorie kirchlicher Praxis an (Innovation).

Wolfgang Marhold kann den größten Teil seiner Ausgangshypothesen empirisch verifizieren und kommt zu dem Schluß, daß es nim Raum der evangelischen Kirche ${ }^{\prime \prime}$... "eine auf Information zur Praxiserhellung und -bewältigung gerichtete Suchhaltung der kirchlichen Funktionsträger gibt" (S. 164), eine Feststellung, die sicherlich im gleichen Maße auf die katholische Kirche zutrifft. Allerdings scheint die Feststellung, die Problematik der gegenwärtigen Selbstauslegung des Christentums werde im Erfahren der Praxis einsichtig, zu optimistisch. Gewiß mag das für den verschwindend geringen Teil derjenigen zutreffen, die die Probleme der Theologie und die der empirischen Sozialforschung kennen. Den Gutwilligen mag ein kleines Buch wie das des Autors helfen, in einzelne Probleme beider Gebiete einzudringen. Die Masse der Betroffenen wird die Praxis dennoch nicht erfahren, solange sich die Theologie nicht ihres Kommunikationsproblems bewußt wird und Konsequenzen zieht.

M.P.B. 
C. J. Medlin: Yearbook Editing, Layout and Management. Ames, Iowa 1966 (Iowa State University Press). 244 Seiten.

Derselbe: Planning your Yearbook. Ames, Iowa 1968 (Iowa State University Press). 128 Seiten.

Schuljahrbücher mit langen Fotoreihen von Graduierten und Lehrern gehören in manchen englischsprechenden Ländern zu jeder Schule und jeder Schülerschaft, die auf sich hält. Das reich mit Beispielen illustrierte Werk von C. J. Medlin gibt ausführliche und praktische Hinweise für die redaktionelle, graphische und auch geschäftliche Arbeit an und mit solchen Jahrbüchern. Die praktische Seite wird besonders in dem Ergänzungsheft "Planning your Yearbook" berücksichtigt, das zu einem großen Teil aus Layout-Blättern besteht, mit denen man vorher eingeführte Übungen durchführen soll. Selbst eine Umrechnungsscheibe für die Bildformate wird mitgeliefert. Dieses Ubungsbuch ist integraler Bestandteil des eigentlichen Handbuches.

Auch wenn vieles in diesem Doppelwerk eben speziell für den nordamerikanischen Markt geschrieben und dargestellt ist, dürfte auch außerhalb der Staaten mancher Verantwortliche für solche oder ähnliche Unternehmungen aus den Büchern Anregungen und Einsichten schöpfen, die er - vielleicht angepaßt - für seine Verhältnisse gebrauchen kann. Gerade weil es im deutschen Raum kaum solche praktischen Handbücher gibt, sei hier besonders auf diese Veröffentlichungen des emeritierten Professors der Kansas State University hingewiesen.

F.J.E.

Roman Muziol: Pressedokumentation. Wegweiser für die Arbeit in Pressearchiven. Mündhen-Pullach/Berlin, 3. erweiterte und ergänzte Auflage 1971 (Verlag Dokumentation). 196 Seiten.

Das Aufgabenfeld Dokumentation hat in den beiden letzten Jahrzehnten immer größere Aufmerksamkeit geweckt: durch das starke Anwachsen der zu dokumentierenden (und also auch zweckmäßig zu archivierenden) Inhalte und durch neu entwickelte Verfahren und technisch-praktische Hilfen, dieser $\mathrm{Pa}$ pierfluten Herr zu werden. Angesichts der Bedeutung, die Veröffentlichungen der publi- zistischen Medien für viele Bereiche des gesellschaftlichen Lebens (nicht zuletzt für die Kirche) gewonnen haben, ist die Pressedokumentation ein neuer Bereich, der in gekonnt, klassisch" geführte Archive nicht so recht passen will. Muziol gibt hier einen auf Erfahrung und theoretischer Kenntnis beruhenden Überblick, der auch als Einführung geeignet ist: Wir könnten uns vorstellen, $\mathrm{da} \beta$ manche diözesane Pressestelle auf die Dauer neben tagesgebundenen Leistungen auch Dokumentationsdienste auf der Basis eines zweckdienlich eingerichteten Pressearchivs zu erbringen haben wird.

S.C.

Walter Nutz: Die Regenbogenpresse. Eine Analyse der deutschen Wochenendblätter. Opladen 1971 (Westdeutscher Verlag, Reihe "WV-Sammlung Soziologie“). 110 Seiten.

Walter Nutz' vom Verlag lange angekündigte Untersuchung über die "Regenbogenpresse $^{\alpha}$ (auch Soraya-Presse genannt) geriet schließlich nur zu einem schmalen Bändchen, dessen Schlußkapitel überdies aus einem vorher veröffentlichten Aufsatz übernommen wurde. Dennoch ein nützliches Bändchen, auch für ein breiteres Publikum, für Lehrer etwa und auch Seelsorger, sofern sie ihre Gemeinden nach und nach soziologisch zu begreifen beginnen. Nutz hat sich als Kenner der alltäglichen Gebrauchsunterhaltungsliteratur ( ${ }^{\text {Trivialliteratur }}{ }^{\prime)}$ mehrfach ausgewiesen. Als Regenbogenpresse untersucht er jetzt jene Wochenend-Unterhaltungsblätter (von der Art "Wochenend", "Sieben Tage“, "Heim und Welt"), die billiger gemacht und billiger im Preis als die Illustrierten in Millionenauflagen unters Lesepublikum gebracht werden; und Nutz verweigert, mindestens als Ausgangsposition, nicht den Schluß: "Was gekauft wird, wird auch gebraucht. “ Seine fünf Grundfragen nach der Thematik der Regenbogenpresse, den in ihr enthaltenen Informationen, nach ihrer "Absidht Traumwelt/Ersatzwelt", nach ihrer Entlastungsfunktion und nach einem von ihr möglicherweise ausgeübten sozialen Harmonisierungseffekt werden dann allerdings verschieden gründlich beantwortet, so daß der Autor selbst am Schluß bekennen muß: „Zu einer intensiveren Erforschung des ungeheuren 
Phänomens der Regenbogenpresse sollte diese Arbeit ein Anstoß sein." In den Teilen, die sich, mehr interpretierend als quantitativ analysierend, mit dem Inhalt jener Blätter befassen, ist sie mehr als ein Anstoß. Der pseudo-märchenartig erzielte Harmonisierungseffekt muß ja nicht nur anti-autoritäre Sorgen im Hinblick auf eine zu wenig fortschrittliche Gesellschaft auslösen, - die pseudo-seelsorgliche Haltung, die aus den hier aufgewiesenen Inhalten der Wochenendpresse deutlich wird, gibt durchaus Anlaß, nach dem sozialpsychischen Zustand einer Gesellschaft (in ganzer Breite) zu fragen, die sidh zu Millionen Tag für Tag und Woche für Woche auf dem Rückzug vor Problemen befindet, die Augen schließt und halbstundenweise unter dem strahlend blauen Himmel des xyz-Kaiserhofes oder Prominenten-swimming-pools wieder zu "sich" kommt.

M.S.

Günther Vogg/Wolfgang Böbm: Massenmedien. Literatur zur Medienpädagogik und politischen Bildung. Düsseldorf und München 1971 (Herausgeber und Bezugsquelle: Landeszentrale für politische Bildung, Düsseldorf, und Wissenschaftliches Institut für Jugend- und Bildungsfragen in Film und Fernsehen, 8 München 15, Waltherstr. 23). 67 Seiten.

Die bisher in vervielfältigter Form zugängliche Bibliographie zur Medienpädagogik und politischen Bildung ist jetzt in gedruckter Form erschienen. Aufgrund ihrer praktikablen systematischen Einteilung ist sie nad. wie vor das beste Hilfsmittel dieser Art, das wir für den Schulgebrauch empfehlen können. Für die Zukunft wären jährliche Nachträge der Neuerscheinungen und vielleicht einmal eine große tierte Bibliographie zu wünschen, - in der jetzigen, beinahe puren Fassung - nur hier und da werden zusätzliche Erklärungen gegeben -, steht Ungleiches und Ungleichwertiges für den Laien kaum erkennbar nebeneinander. Die Einteilung des nützlichen Bändchens ist folgende: I. Umfassende und theoretische Arbeiten, II. Spezielle Darstellungen, III. Film- und Fernsehkunde, IV. Information und politische Meinungsbildung im Bereich der Massenmedien, V. Massen- medien und Politik aus der Sicht verschiedener Gesellschaftssysteme, VI. Massenmedien und politische Bildung, VII. Kirchliche Film- und Fernseharbeit, VIII. Ausländische Literatur, IX. Sammelhefte, Handbücher, Lexika (hier fehlt das "Handbuch der Weltpresse", Opladen 1970), X. Bibliographien, XI. Zeitschriften, XII. Presse- und Informationsdienste, XIII. Verzeichnisse über Filme, Bildreihen, Tonträger.

M. S.

\section{ZEITSCHRIFTENHINWEISE}

\section{Allgemeine Publizistik}

Stanley Nyabwa, Multimedia Zambia. In: „WACC-Journal“, Witten/Ruhr 2:1971 (Heft 1), S. 25-28.

\section{Presse}

Albert Oscar Bolela, Un aperçu de la presse congolaise écrite par les noirs de 1885 à 1960. In: „Congo-A frique ${ }^{\text {, }}$, Kinshasa 11:1971 (Janvier), S. 5-23.

Verfasser ist kongolesischer Botschafter in Schweden und Mitglied verschiedener internationaler Journalistenverbände.

- Katholische Presse als Streitobjekt. Verschiedene Beiträge von einer Tagung der Katholischen Akademie in Bayern (26. bis 28. 2.1971). Autoren: Frederico Alessandrini, Luitpold A. Dorn, Ferdinand Oertel, Mario von Galli, Joachim Westerbarkey. In: „Zur Debatte, Themen der Katholischen Akademie in Bayern", Mündhen 1:1971 (Nr. 3, März), S. 1-6.

\section{Hörfunk}

Sigurd Aske, Radio Voice of the Gospel, Political, Cross-Cultural and Theological Aspects. In: "WACC-Journal“, Witten/Ruhr 2:1971 (Heft 1), S. 16-18. 
Cardinal Gray, Pastoral aspects of Radio and Television. In: „UNDA, Revue catholique de radio et de television 1971“, No. 1, S. $8-11$.

Ansprache des Kardinals von Edinburgh bei der UNDA-Generalversammlung in New Orleans.

Heinz Linnerz, Ưberlegungen zum Verhältnis Kirchen - Rundfunk. In: "Medium“, München $8: 1971$, S. 24-27.

Aus einem Gespräch mit Vertretern der Kirchen in Essen am 15. 12. 1970.

Edwin Robertson, Christian Broadcasting in Africa. In: „WACC-Journal“, Witten/Ruhr 2:1971 (Heft 1), S. 10.

Fernsehen

E. Victor Badejo, Radio and Television Credibility. In: "WACC-Journal", Witten/ Ruhr 2:1971 (Heft 1), S. 23-24.

Enrico Baragli, Verso una riforma della RAI-TV. In: "La Civiltà Cattolica“, Roma 122:1971, I, S. 7-17; S. 140-148 (Quaderni 2893-2894).
Jörg Zink, Das Wort zum Sonntag, Wie spricht man zu Zeitgenossen? In: „Medium“, München 8:1971, S. 9-23.

Vortrag bei der Sprechertagung der Schweizer Kirchen am 19. 10. 1970.

Bernbard Grom, Kurzfilme in der religiösen Jugend- und Erwachsenenbildung. In: ${ }_{n}$ Stimmen der Zeit", München 96:1971, S. 256 bis 270 .

Rudolf Joos, Aspekte zukünftiger evangelischer Filmarbeit. In: "Medium“, München $8: 1971$, S. 51-56.

\section{Sonstige Gebiete}

Peter Kapitza, Schaufenster Gottes, Zur Funktion und Sprache des Buchtitels in der gegenwärtigen religiösen Literatur. In: ${ }_{n}$ Stimmen der Zeit" ${ }^{4}$, München 96:1971, S. 217 bis 235 .

Norbert Mette, Offentlichkeitsarbeit in der Kirche. In: "Diakonia - Der Seelsorger", Mainz-Wien 2:1971 (Heft 2), S. 110-114. 